Supporting information

\title{
Methylation Design Strategy to Trigger a Dual Dielectric Switch and Improve the Phase Transition Temperature
}

Jia Wang ${ }^{\dagger}$, , Tie Zhang ${ }^{\dagger}$, Zhi-Xu Zhang ${ }^{\dagger}$, Chang-Yuan Su ${ }^{\dagger}$, Yi Zhang ${ }^{*}{ }^{\dagger}$ and Da-Wei Fu ${ }^{*}$, (†Ordered Matter Science Research Center, Jiangsu Key Laboratory for Science and Applications of Molecular Ferroelectrics, Southeast University, Nanjing, 211189, P.R. China

§Institute for Science and Applications of Molecular Ferroelectrics, Department of Chemistry, Zhejiang Normal University, Jinhua, 321004, P.R. China)

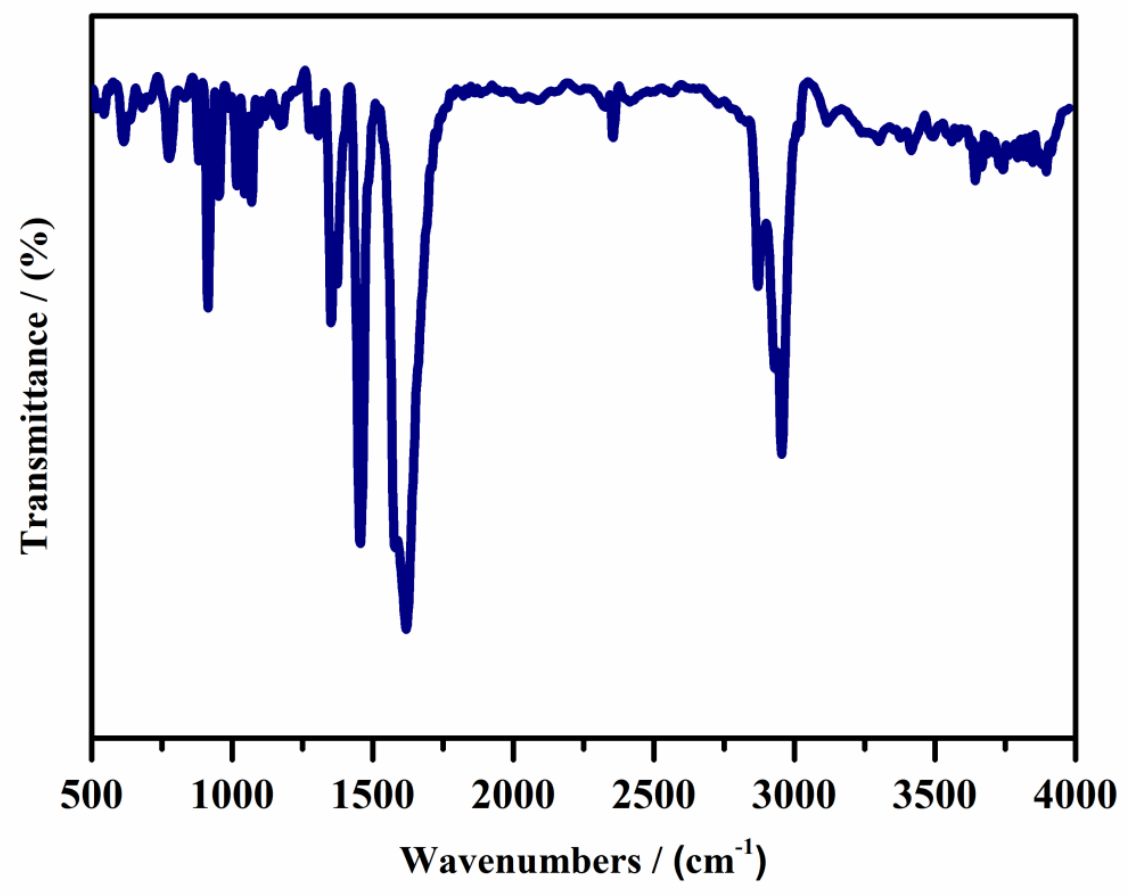

Fig. S1 The IR spectrum of compound $\mathbf{1}$. 

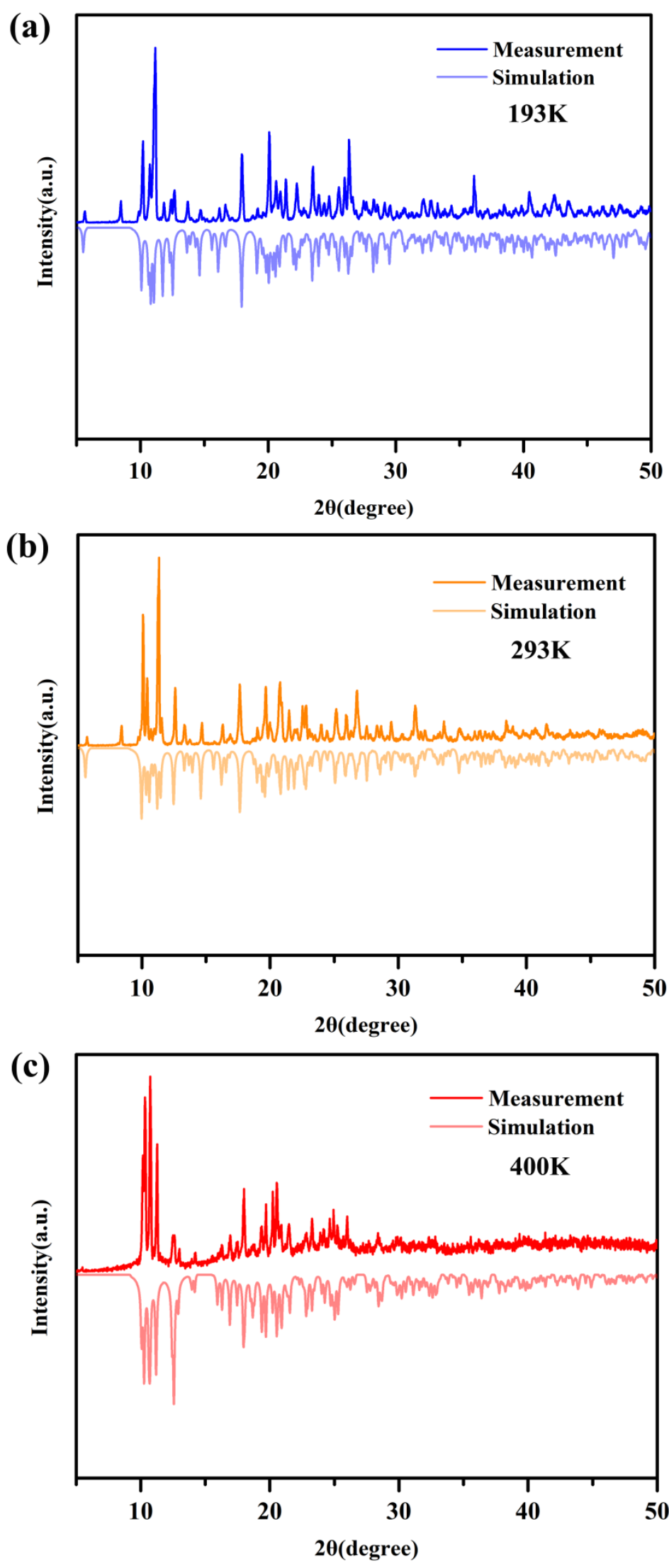

Fig. S2 The experimental and simulated PXRD patterns of compound $\mathbf{1}$ at (a) $193 \mathrm{~K}$, (b) $293 \mathrm{~K}$ and (c) $400 \mathrm{~K}$. 


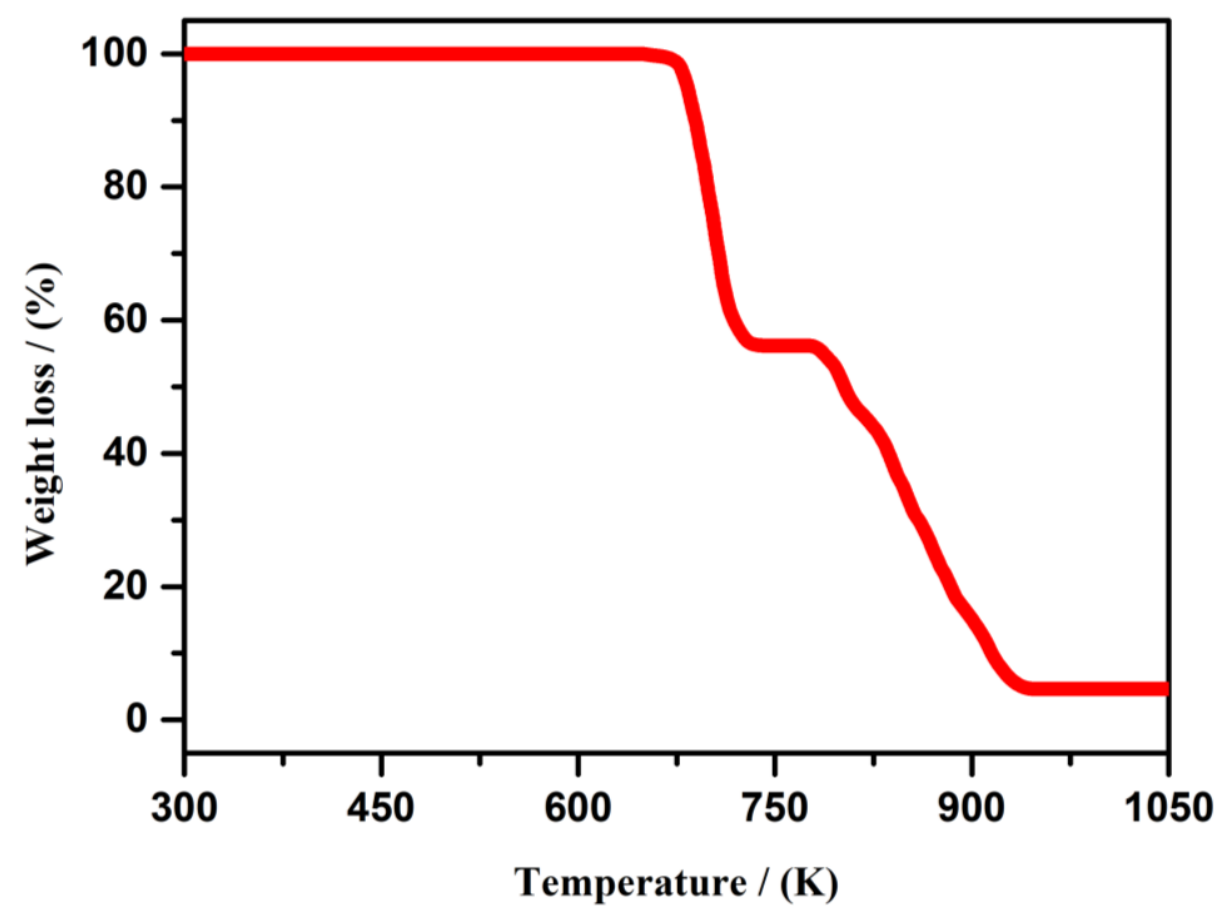

Fig. S3 TGA curve of compound 1.

Table S1. Crystal data and structure refinements for 1 at 193K, 293K and 400K.

\begin{tabular}{cccc}
\hline & $\mathbf{L T P}(\mathbf{1 9 3 K})$ & $\mathbf{R T P}(\mathbf{2 9 3 K})$ & $\mathbf{H T P}(\mathbf{4 0 0 K})$ \\
\hline Empirical formula & $\mathrm{C}_{20} \mathrm{H}_{40} \mathrm{~N}_{2} \mathrm{CdCl}_{4}$ & $\mathrm{C}_{20} \mathrm{H}_{40} \mathrm{~N}_{2} \mathrm{CdCl}_{4}$ & $\mathrm{C}_{20} \mathrm{H}_{40} \mathrm{~N}_{2} \mathrm{CdCl}_{4}$ \\
$\mathbf{M}_{\mathbf{r}}$ & 562.75 & 562.75 & 562.75 \\
Temperature/ K & 193 & 293 & 400 \\
Crystal system & monoclinic & monoclinic & monoclinic \\
Space group & $\mathrm{P} 21 / \mathrm{c}$ & $\mathrm{P} 21 / \mathrm{c}$ & $\mathrm{P} 21 / \mathrm{c}$ \\
$\mathbf{a} / \AA$ & $16.2307(4)$ & $16.1654(11)$ & $17.324(3)$ \\
b/ $\AA$ & $9.2494(2)$ & $9.4675(6)$ & $9.4934(18)$ \\
c/ $\AA$ & $17.7959(5)$ & $18.1411(10)$ & $18.027(4)$ \\
$\mathbf{\alpha} /{ }^{\circ}$ & 90 & 90 & 90
\end{tabular}




\begin{tabular}{cccc}
$\boldsymbol{\beta} /{ }^{\circ}$ & $99.805(2)$ & $102.451(6)$ & $107.23(2)$ \\
$\boldsymbol{\gamma} /{ }^{\circ}$ & 90 & 90 & 90 \\
Volume/ Å3 & $2632.57(11)$ & $2711.1(3)$ & $2831.7(10)$ \\
$\mathbf{Z}$ & 4 & 4 & 4 \\
Radiation type & Mo-Ka & Mo-Ka & Mo-Ka \\
Absorption & Semi-empirical & Semi-empirical & Semi-empirical \\
correction & & & \\
Dcalc / g cm-3 & 1.108 & 1.379 & 1.225 \\
$\mathbf{F}(\mathbf{0 0 0})$ & 1160 & 1160 & 1000 \\
$\mathbf{G O F}$ & 1.509 & 1.129 & 0.923 \\
R1[I > 26(I)] & 0.1445 & 0.1697 & 0.2065 \\
wR2[I > 26(I)] & 0.3465 & 0.5045 & 0.4826 \\
\hline
\end{tabular}

Table S2. Selected bond lengths $[\AA]$ for compound 1 at $193 \mathrm{~K}$.

\begin{tabular}{ccccc}
\hline Temperature & \multicolumn{2}{c}{ Bond length $(\mathbf{\AA})$} & \multicolumn{2}{c}{ Bond angles $\left[{ }^{\circ}\right]$} \\
\hline $193 \mathrm{~K}$ & $\mathrm{Cd} 1-\mathrm{Cl1}$ & $2.545(2)$ & $\mathrm{Cl} 1-\mathrm{Cd} 1-\mathrm{Cl} 2$ & $109.66(6)$ \\
& $\mathrm{Cd} 1-\mathrm{Cl} 2$ & $2.5946(16)$ & $\mathrm{Cl} 1-\mathrm{Cd} 1-\mathrm{Cl} 3$ & $111.90(7)$ \\
& $\mathrm{Cd} 1-\mathrm{Cl} 3$ & $2.552(2)$ & $\mathrm{Cl} 1-\mathrm{Cd} 1-\mathrm{Cl} 4$ & $108.03(7)$ \\
& $\mathrm{Cd} 1-\mathrm{Cl} 14$ & $2.547(2)$ & $\mathrm{Cl}-\mathrm{Cd} 1-\mathrm{Cl} 2$ & $113.16(6)$ \\
& & & $\mathrm{Cl} 1-\mathrm{Cd} 1-\mathrm{Cl} 2$ & $107.19(7)$ \\
& & & $\mathrm{Cl} 1-\mathrm{Cd} 1-\mathrm{Cl} 3$ & $106.62(8)$ \\
\hline
\end{tabular}

Table S3. Selected bond lengths $[\AA ̊]$ for compound 1 at $293 \mathrm{~K}$.

\begin{tabular}{clrll}
\hline Temperature & \multicolumn{2}{c}{ Bond length $(\AA)$} & \multicolumn{2}{c}{ Bond angles $\left[{ }^{\circ}\right]$} \\
\hline \multirow{2}{2}{$93 \mathrm{~K}$} & $\mathrm{Cd} 1-\mathrm{Cl} 1$ & $2.551(3)$ & $\mathrm{C} 12-\mathrm{Cd} 1-\mathrm{Cl} 1$ & $111.85(10)$ \\
& $\mathrm{Cd} 1-\mathrm{Cl} 2$ & $2.548(3)$ & $\mathrm{Cl} 2-\mathrm{Cd} 1-\mathrm{Cl} 3$ & $109.70(10)$ \\
& $\mathrm{Cd} 1-\mathrm{Cl} 3$ & $2.579(3)$ & $\mathrm{C} 12-\mathrm{Cd} 1-\mathrm{C} 14$ & $107.97(11)$
\end{tabular}




\begin{tabular}{llll}
$\mathrm{Cd} 1-\mathrm{Cl} 4$ & $2.559(3)$ & $\mathrm{Cl} 1-\mathrm{Cd} 1-\mathrm{Cl} 3$ & $110.99(13)$ \\
& & $\mathrm{Cl}-\mathrm{Cd} 1-\mathrm{Cl} 4$ & $109.45(15)$ \\
& $\mathrm{Cl}-\mathrm{Cd} 1-\mathrm{Cl} 3$ & $106.71(12)$ \\
\hline
\end{tabular}

Table S4. Selected bond lengths $[\AA]$ for compound 1 at $400 \mathrm{~K}$.

\begin{tabular}{ccccc}
\hline Temperature & \multicolumn{2}{c}{ Bond length $(\mathbf{\AA})$} & \multicolumn{2}{c}{ Bond angles $\left[{ }^{\circ}\right]$} \\
\hline $400 \mathrm{~K}$ & $\mathrm{Cd} 1-\mathrm{Cl1}$ & $2.539(4)$ & $\mathrm{Cl} 1-\mathrm{Cd} 1-\mathrm{Cl} 2$ & $108.54(12)$ \\
& $\mathrm{Cd} 1-\mathrm{Cl} 2$ & $2.570(4)$ & $\mathrm{Cl}-\mathrm{Cd} 1-\mathrm{C} 11$ & $104.49(13)$ \\
& $\mathrm{Cd} 1-\mathrm{Cl3}$ & $2.519(4)$ & $\mathrm{Cl} 1-\mathrm{Cd} 1-\mathrm{C} 22$ & $111.45(15)$ \\
& $\mathrm{Cd} 1-\mathrm{Cl} 14$ & $2.457(5)$ & $\mathrm{Cl} 1-\mathrm{Cd} 1-\mathrm{C} 11$ & $110.92(18)$ \\
& & & $\mathrm{Cl} 1-\mathrm{Cd} 1-\mathrm{Cl} 2$ & $107.28(12)$ \\
& & & $\mathrm{Cl} 1-\mathrm{Cd} 1-\mathrm{Cl} 3$ & $114.1(2)$ \\
\hline
\end{tabular}

